\title{
Daikenchuto, a Traditional Kampo Medicine, Facilitates Mucosal Hyperemic Responses through Activation of TRPA1- and TRPV1- Expressing Sensory Nerves in Rat Stomach
}

\section{(๑) $\odot \Theta$}

\author{
Authors

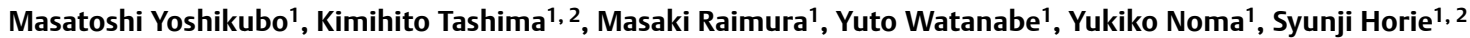

\section{Affiliations}

1 Laboratory of Pharmacology, Faculty of Pharmaceutical Sciences, Josai International University, Togane, Chiba, Japan

2 Center of Pharmacological Kampo Research, Josai International University, 1 Gumyo, Togane, Chiba, Japan

Key words

daikenchuto, gastric mucosal blood flow, TRPA1, TRPV1, sensory nerves, rat

$$
\begin{array}{ll}
\text { received } & 10.04 .2021 \\
\text { revised } & 23.09 .2021 \\
\text { accepted } & 19.10 .2021
\end{array}
$$

\section{Bibliography}

Planta Med Int Open 2022; 9: e72-e79

DOI 10.1055/a-1712-8391

ISSN 2509-9264

(c) 2022. The Author(s).

This is an open access article published by Thieme under the terms of the Creative Commons Attribution-NonDerivative-NonCommercial-License, permitting copying and reproduction so long as the original work is given appropriate credit. Contents may not be used for commercial purposes, or adapted, remixed, transformed or built upon. (https://creativecommons. org/licenses/by-nc-nd/4.0/)

Georg Thieme Verlag KG, Rüdigerstraße 14,

70469 Stuttgart, Germany

\section{Correspondence}

Kimihito Tashima, PhD

Laboratory of Pharmacology

Faculty of Pharmaceutical Sciences

Josai International University

1 Gumyo

Togane

283-8555 Chiba

Japan

Tel.: + 81/475/53 4585, Fax: + 81/475/55 8811

ktashima@jiu.ac.jp $\circledast$ Supplementary material is available under https://doi.org/10.1055/a-1712-8391.

\section{ABSTRACT}

Daikenchuto is a traditional Kampo medicine used to treat cold sensations and dysmotility in the abdomen. This study investigated the roles of transient receptor potential ankyrin 1 - and transient reporter potential vanilloid 1 -expressing sensory nerves in daikenchuto-induced gastric mucosal blood flow by pharmacological and immunohistochemical analyses using male SpragueDawley rats. Gastric mucosal blood flow was measured in ex vivo stomachs using a laser Doppler flowmeter. Transient receptor potential ankyrin 1 activator allyl isothiocyanate or transient reporter potential vanilloid 1 activator capsaicin were applied for $10 \mathrm{~min}$, and daikenchuto was applied for 10 or $30 \mathrm{~min}$ to the rat stomach. Transient reporter potential vanilloid 1 blocker $\mathrm{N}$-(4-tbutylphenyl)-4-(3-chlopyridin-2-yl) tetrahydropyrazine-1(2H)carboxamide and transient receptor potential ankyrin 1 blocker A-967079 were also administered intragastrically. Capsaicin and allyl isothiocyanate increased gastric mucosal blood flow immediately after the intragastric application, which was almost completely inhibited by $\mathrm{N}$-(4-t-butylphenyl)-4-(3-chlopyridin-2-yl) tetrahydropyrazine-1(2H)-carboxamide and A-967079, respectively. Daikenchuto increased gastric mucosal blood flow in a concentration-dependent manner. A-967079 significantly inhibited the increase in gastric mucosal blood flow induced by daikenchuto. In contrast, $N$-(4-t-butylphenyl)-4-(3-chlopyridin-2-yl) tetrahydropyrazine-1(2H)-carboxamide inhibited the responses to daikenchuto only in the late phase but not in the initial phase. Interestingly, in the deafferentation of capsaicin-sensitive sensory fiber in rats, the increased gastric mucosal blood flow induced by daikenchuto was only decreased in the late phase. Although transient receptor potential ankyrin 1 - and transient reporter potential vanilloid 1 -immunoreactive fibers were observed around the submucosal blood vessels of normal subjects, they were completely absent in the deafferentation of capsaicin-sensitive sensory fibers. Thus, daikenchuto increases gastric mucosal blood flow via transient receptor potential ankyrin 1- and transient reporter potential vanilloid 1-co-expressing sensory nerves in rat stomachs. 


\section{ABBREVIATIONS \\ BCTC N-(4-t-butylphenyl)-4-(3-chlopyridin-2-yl) \\ tetrahydropyrazine-1(2H)-carboxamide \\ CGRP calcitonin gene-related peptide \\ CMC carboxymethylcellulose \\ DKT daikenchuto \\ GMBF gastric mucosal blood flow \\ TRPA1 transient receptor potential ankyrin 1 \\ TRPV1 transient reporter potential vanilloid 1}

\section{Introduction}

DKT, one of Japan's most frequently prescribed Kampo formulas, decreases intestinal dysmotility and inflammation and cures cold sensations in the abdomen, mainly in the umbilical sections [1,2]. DKT comprises the following crude ingredients: processed ginger, ginseng, zanthoxylum fruit, and malt sugar. DKT increases blood flow in the superior mesenteric arteries in human and rat intestinal mucosa $[3,4]$, and it improves small intestinal motility in guinea pigs by activating the TRPV1 [5,6]. A previous study has shown that DKT increases the levels of gastrointestinal hormones and neuropeptides such as motilin, vasoactive intestinal peptide, and CGRP [7]. Additionally, DKT increases mucosal blood flow in the rat colon by upregulating adrenomedullin and activating the TRPA 1 in the epithelium $[4,8]$. These findings suggest that an increase in mucosal blood flow in the gastrointestinal tract is responsible for the clinical effects of DKT against cold sensations and dysmotility in the abdomen. However, the underlying mechanism for the activation of TRPA 1 and TRPV 1 in sensory nerves that leads to an increase in DKT-induced GMBF has not been elucidated yet. This study aimed to determine whether DKT facilitates an increase in GMBF in the rat stomach through activation of TRPV1- and TRPA1-expressing sensory nerves.

\section{Results}

First, we studied the vasoactive effects of TRPV1 and TRPA1 activators on rat gastric mucosa. The TRPV1 activator capsaicin $(10 \mathrm{mmol} / \mathrm{L})$ induced an increase in GMBF, which peaked after 5 min of mucosal application of capsaicin and remained at sub-maximum levels until 15 min; following this, the responses returned to basal levels ( $\triangleright$ Fig. 1a). The TRPA1 activator allyl isothiocyanate (AITC, $10 \mathrm{mmol} / \mathrm{L}$ ) induced a rapid increase in GMBF (251.1 $\pm 20.2 \%)$, which gradually decreased to baseline values after 30 min of application ( $\$$ Fig. 2a). The TRPV1 blocker $\mathrm{BCTC}(2.7 \mathrm{mmol} / \mathrm{L})$ clearly inhibited $\mathrm{GMBF}$ in response to capsaicin; however, the TRPA1 blocker A-967079 (10 mmol/L) did not show similar effects (- Fig. 1). In contrast, the TRPA1 blocker A-967079 (10 mmol/L), but not BCTC, completely suppressed GMBF in response to AITC ( $>$ Fig. 2). These blockers had no activity of their own.

Intragastric administration of DKT $(360,720$, and $1440 \mathrm{mg} / \mathrm{mL})$ induced gastric hyperemic responses in a concentration-dependent manner, and a significant effect was observed at concentrations of $720 \mathrm{mg} / \mathrm{mL}$ or higher ( $\vee$ Fig. 3a). The maximal responses of GMBF to 10 min of intragastric application of DKT at 360,720 , and $1440 \mathrm{mg} /$ $\mathrm{mL}$ were $128.9 \pm 6.2 \%, 136.0 \pm 10.4 \%$, and $162.9 \pm 8.5 \%$, respective-

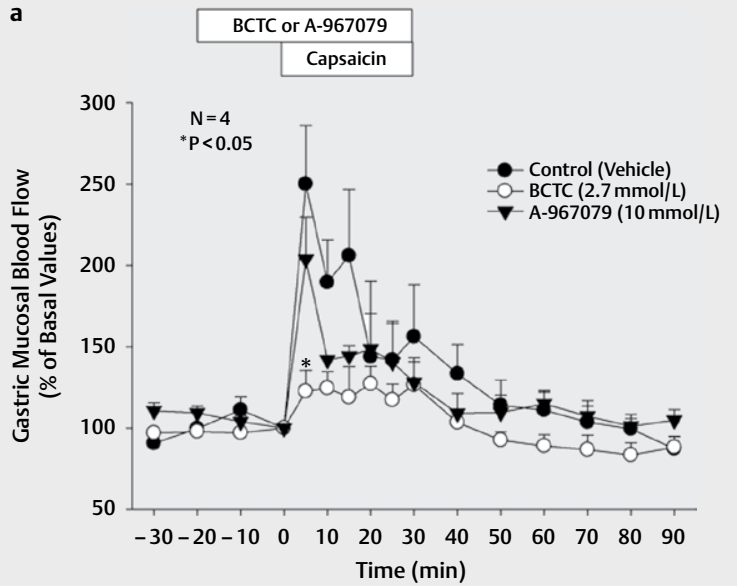

b

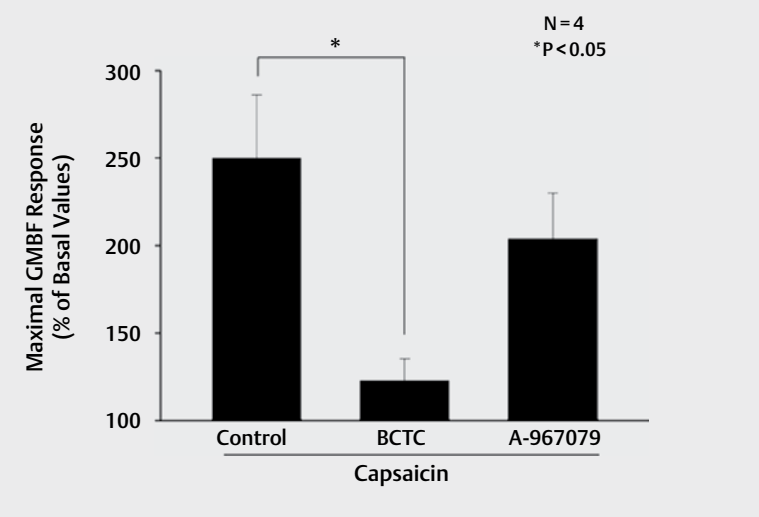

- Fig. 1 Effects of TRPV1 blocker BCTC and TRPA1 blocker A -967079 on GMBF induced by the mucosal application of capsaicin in rat stomachs. (a) Time course of GMBF response to capsaicin $(10 \mathrm{mmol} / \mathrm{L})$ in rat stomachs is shown. BCTC $(2.7 \mathrm{mmol} / \mathrm{L})$ was applied to the chamber for $50 \mathrm{~min}$ starting at $20 \mathrm{~min}$ prior to capsaicin $(10 \mathrm{mmol} / \mathrm{L})$ application. Capsaicin was topically applied to the mucosa for $30 \mathrm{~min}$. The data are expressed as a \% increase in basal values and represented as mean \pm S.E. of values every 5 or $10 \mathrm{~min}$ for 4 rats. ${ }^{*} p<0.05$ was considered statistically significant when compared to the control group using Student's t-test. (b) Maximal GMBF response induced by the mucosal application of capsaicin $(10 \mathrm{mmol} / \mathrm{L})$ in the presence of BCTC $(2.7 \mathrm{mmol} / \mathrm{L})$ or A-967079 $(10 \mathrm{mmol} / \mathrm{L})$. The data are expressed as a \% increase in basal values and represented as mean \pm S.E. for 4 rats. ${ }^{*} p<0.05$ was considered statistically significant when compared to the control group using Student's t-test. Note that an increased GMBF in response to capsaicin $(10 \mathrm{mmol} / \mathrm{L})$ was almost completely inhibited by BCTC ( $2.7 \mathrm{mmol} / \mathrm{L})$ but not A-967079 (10 mmol/L).

ly ( $\triangleright$ Fig. 3b). Mucosal application of the vehicle (distilled water) did not increase GMBF ( $\triangleright$ Fig. $3 \mathbf{a}, \mathbf{b}$ ). GMBF reached its maximum value after administration of $1440 \mathrm{mg} / \mathrm{mL}$ of DKT; thus, this concentration was used in subsequent experiments to examine the effects of various agents on GMBF in response to DKT. Interestingly, the maximum GMBF levels after administration of $1440 \mathrm{mg} / \mathrm{mL}$ of DKT were similar to that observed after administration of capsaicin $(1 \mathrm{mmol} / \mathrm{L})$ into the rat stomach (Supporting Information Fig. 1b). 


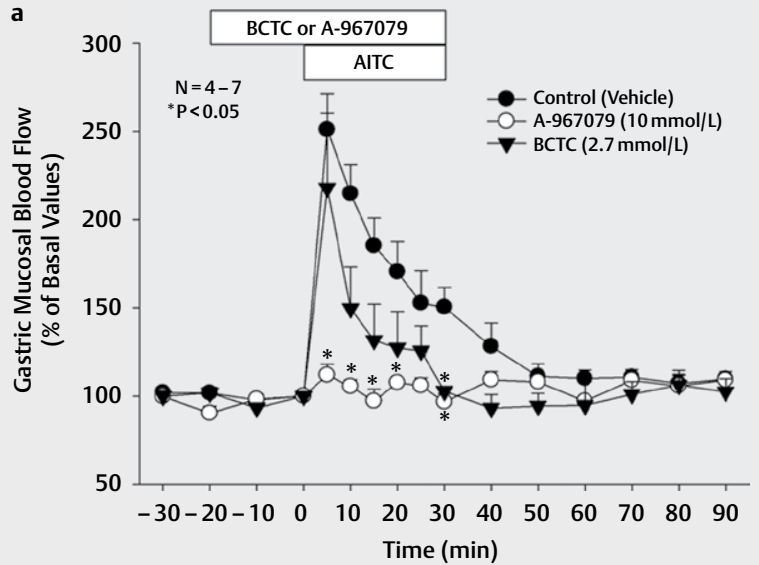

b

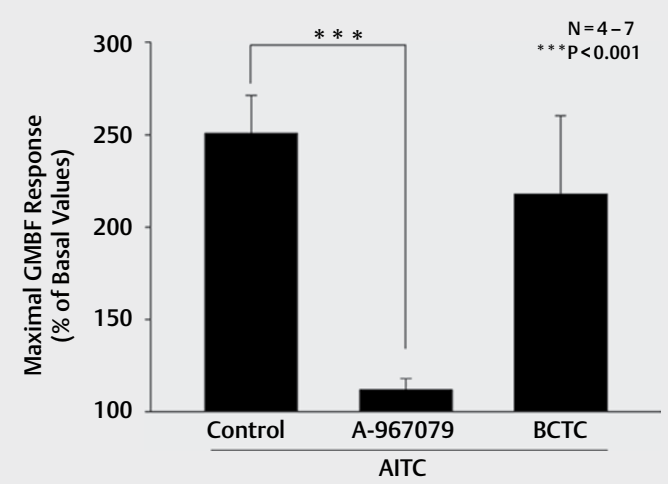

- Fig. 2 Effects of TRPA1 blocker A-967079 and TRPV1 blocker BCTC on gastric mucosal blood flow (GMBF) induced by the mucosal application of allyl isothiocyanate (AITC) in rat stomachs. (a) Time course of GMBF response to AITC $(10 \mathrm{mmol} / \mathrm{L})$ in rat stomachs is shown. A-967079 $(10 \mathrm{mmol} / \mathrm{L})$ was applied to the chamber for $50 \mathrm{~min}$ starting at $20 \mathrm{~min}$ prior to AITC $(10 \mathrm{mmol} / \mathrm{L})$ application. AITC was topically applied to the mucosa for $30 \mathrm{~min}$. The data are expressed as a \% increase in basal values, and they represent mean \pm S.E. of values every 5 or 10 min for $4-7$ rats. ${ }^{*} p<0.05$ was considered statistically significant using Student's t-test. (b) Maximal GMBF response induced by mucosal application of AITC $(10 \mathrm{mmol} / \mathrm{L})$ in the presence of BCTC $(2.7 \mathrm{mmol} / \mathrm{L})$ or A-967079 $(10 \mathrm{mmol} / \mathrm{L})$. The data are expressed as $\mathrm{a} \%$ increase in basal values and represented as mean \pm S.E. for $4-7$ rats. ${ }^{* *}{ }^{*} \mathrm{p}<0.001$ was considered statistically significant compared to the control group using Student's t-test. Note that an increased GMBF in response to AITC $(10 \mathrm{mmol} / \mathrm{L})$ was completely inhibited by A-967079 (10 mmol/L) but not BCTC (2.7 mmol/L).

Intragastric pre-application of the TRPV1 blocker BCTC (2.7 mmol/L) alone did not affect GMBF. The mucosal application time of DKT $(1440 \mathrm{mg} / \mathrm{mL})$ was extended from 10 to $30 \mathrm{~min}$ to investigate further the effects of initial and late periods during DKT application in rats ( $\mathbf{F i g}$. 4a). A significant increase in GMBF was observed in response to DKT $(1440 \mathrm{mg} / \mathrm{mL})$ for $30 \mathrm{~min}$, and the gastric hyperemic responses induced by DKT $(1440 \mathrm{mg} / \mathrm{mL})$ after 10 and 30 min of application were $216.5 \pm 15.4 \%$ and $178.0 \pm 20.1 \%$, respectively ( $>$ Fig. $\mathbf{4 b}, \mathbf{c}$ ). The increase in GMBF in response to DKT $(1440 \mathrm{mg} / \mathrm{mL})$ was decreased by BCTC $(2.7 \mathrm{mmol} / \mathrm{L})$ during the late period but not during the initial period. The values of GMBF after 10

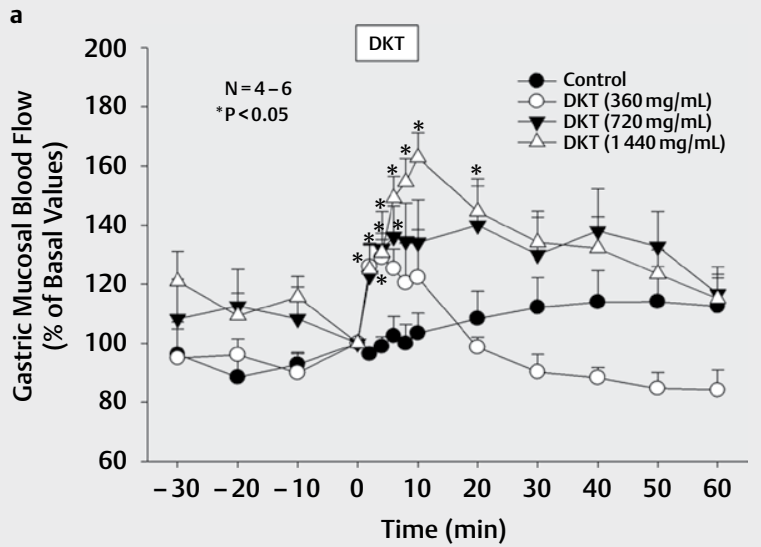

b

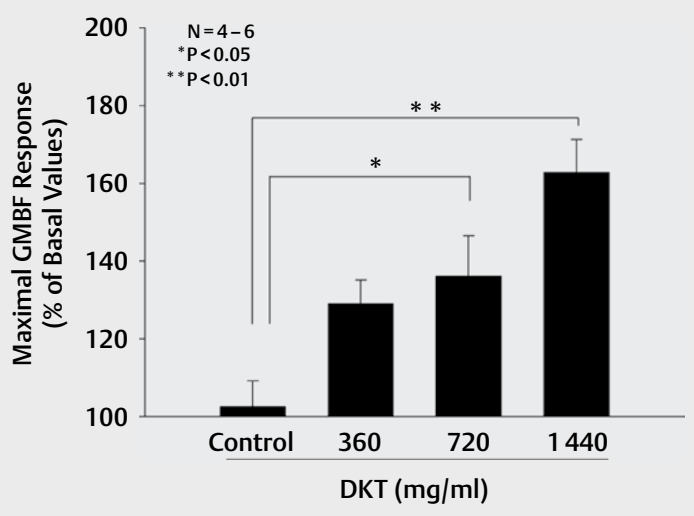

- Fig. 3 Effect of the mucosal application of daikenchuto (DKT: 360,720 , and $1440 \mathrm{mg} / \mathrm{mL}$ ) on GMBF in rat stomachs. (a) Time course of GMBF response to DKT. The data are expressed as a \% increase in baseline values, and they represent the mean $\pm \mathrm{SE}$ obtained every 2 or 10 min for $4-6$ rats. ${ }^{*} p<0.05$ was considered statistically significant compared to the control group using ANOVA with Dunnett's test. (b) Maximum response of GMBF induced by the mucosal application of DKT $(360,720$, and $1440 \mathrm{mg} / \mathrm{mL})$. The data are expressed as a $\%$ increase in baseline value and represented as mean \pm SE for $4-6$ rats. ${ }^{*} \mathrm{P}<0.05$ and ${ }^{*}{ }^{*} \mathrm{p}<0.01$ were considered statistically significant compared to the control group using ANOVA with Dunnet's test. Note that GMBF response to DKT increases in a concentration-dependent manner.

and 30 min of mucosal application of DKT in the presence of BCTC ( $2.7 \mathrm{mmol} / \mathrm{L}$ ) were $203.3 \pm 34.3 \%$ and $150.6 \pm 5.3 \%$, respectively. In contrast, the increase in GMBF in response to DKT $(1440 \mathrm{mg} / \mathrm{mL})$ in the initial and late periods was decreased by the TRPA1 blocker A-967079 (10 mmol/L) (> Fig. 4a). The GMBF values after 10 and 30 min of mucosal application of DKT in the presence of A-967079 $(10 \mathrm{mmol} / \mathrm{L})$ were $138.3 \pm 11.1 \%$ and $139.1 \pm 14.3 \%$, respectively ( Fig. 4b, c). Intragastric pre-application of A-967079 (10 mmol/L) alone did not affect GMBF. These results suggest that intragastric DKT induces a significant increase in GMBF via activation of TRPV1 and TRPA1, which may be due to the gastric epithelial cell expressing TRPA 1 in the initial phase followed by the TRPA 1 - and TRPV1 coexpressing nerve fiber in the late phase.

As expected, it was observed that the increased GMBF in response to DKT was inhibited by the TRPA 1 blocker A967079 in both 

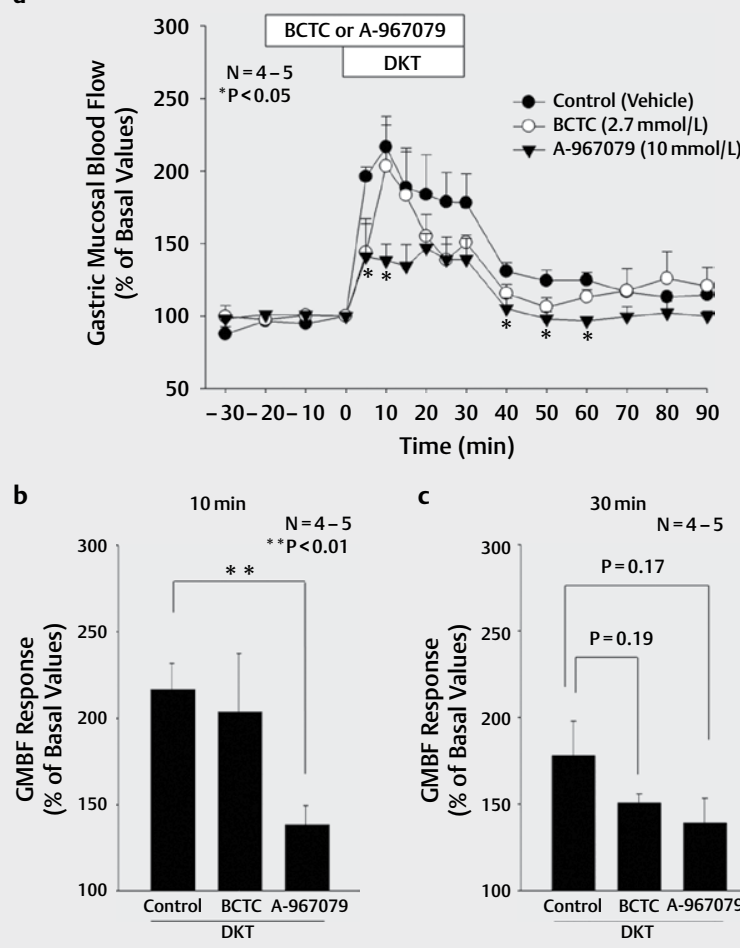

c

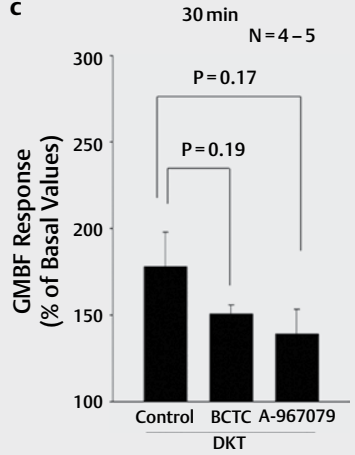

Fig. 4 Effect of TRPV1 blocker BCTC and TRPA1 blocker A-967079 on GMBF induced by the mucosal application of DKT in rat stomachs. (a) Time course for analysis of GMBF response to DKT $(1440 \mathrm{mg} / \mathrm{mL})$ in rat stomachs treated with either A-967079 $(10 \mathrm{mmol} / \mathrm{L})$ or BCTC $(2.7 \mathrm{mmol} / \mathrm{L})$. The data are expressed as a \% increase in baseline values, and they represent the mean \pm SE values obtained every 5 or 10 min for $4-5$ rats. ${ }^{*} \mathrm{P}<0.05$ was compared with the vehicle using Student's t-test. (b) GMBF after 10 min of DKT administration $(1440 \mathrm{mg} / \mathrm{mL})$ in rat stomachs treated with either A-967079 $(10 \mathrm{mmol} / \mathrm{L})$ or BCTC ( $2.7 \mathrm{mmol} / \mathrm{L})$. (c) GMBF after $30 \mathrm{~min}$ of DKT $(1440 \mathrm{mg} / \mathrm{mL})$ administration in rat stomachs treated with either A-967079 (10 mmol/L) or BCTC ( $2.7 \mathrm{mmol} / \mathrm{L})$. The data are expressed as a $\%$ increase in baseline values and represented as mean \pm SE for 4-5 rats. ${ }^{* *} p<0.01$ was compared with the vehicle using Student's t-test. Note that the increased GMBF in response to DKT is obviously suppressed by A-967079 but is not partially suppressed by BCTC in the initial periods.

initial and late phases, while the TRPV1 blocker BCTC inhibited the increased GMBF in response to DKT only in the late phase of the experiment.

A significant increase in GMBF in response to DKT $(1440 \mathrm{mg} / \mathrm{mL})$ for 30 min was observed in animals treated with the vehicle (distilled water), and the gastric hyperemic responses induced by DKT $(1440 \mathrm{mg} / \mathrm{mL})$ after 10 and $30 \mathrm{~min}$ of application were $144.5 \pm 31.6 \%$ and $143.0 \pm 10.4 \%$, respectively ( $\triangleright$ Fig. 5 a). The increase in GMBF values in response to mucosal application of DKT $(1440 \mathrm{mg} / \mathrm{mL})$ in the deafferentation of capsaicin-sensitive sensory fibers were $122.5 \pm 11.1 \%$ and $103.4 \pm 6.8 \%$, after 10 and 30 min, respectively, implying a significant decrease in the late phase, but not in the initial phase ( $\mathbf{F i g}$. 5b, c). GMBF response to DKT after removing mucosal application was similar to that observed in BCTC ( $2.7 \mathrm{mmol} / \mathrm{L})$-treated a

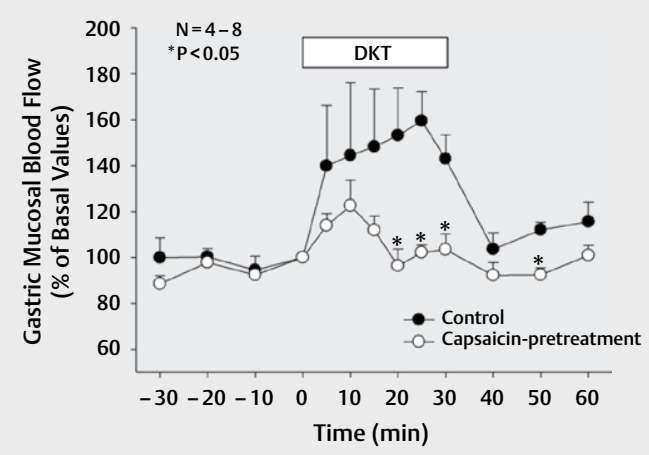

b
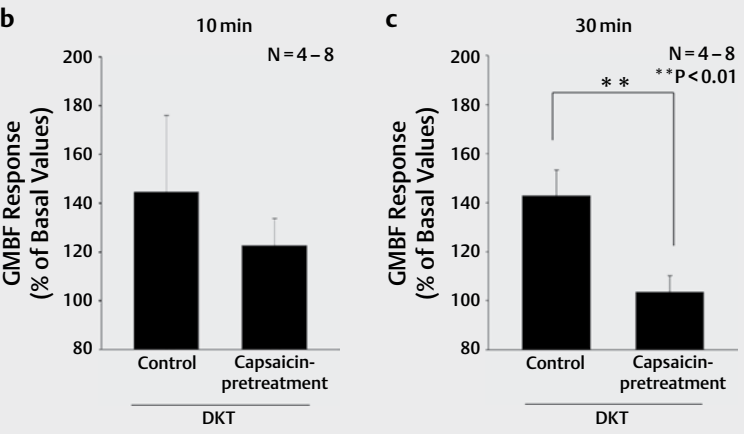

- Fig. 5 Effect of deafferentation of capsaicin-sensitive sensory fiber on GMBF induced by the mucosal application of DKT in rat stomachs. DKT $(1440 \mathrm{mg} / \mathrm{mL})$ was applied topically to the mucosa for $30 \mathrm{~min}$, and the stomach was perfused with saline before and after application. (a) Time course for analysis of $G M B F$ in response to DKT $(1440 \mathrm{mg} / \mathrm{mL})$ in capsaicin-pretreated rats. Data are expressed as the \% increase in baseline values and represented as mean \pm SE values obtained every 5 or 10 min for $4-8$ rats. ${ }^{*} p<0.05$ was considered statistically significant compared to the control group using Student's t-test. b) and c) GMBF after 10 and 30 min of mucosal application of DKT $(1440 \mathrm{mg} / \mathrm{mL})$ in capsaicin-pretreated rats, respectively. Data are expressed as the \% increase in baseline values and represented as mean \pm SE for $4-8$ rats. ${ }^{*} \mathrm{p}<0.01$ was considered statistically significant when they compared to the control group using Student's t-test.

animals as shown in $>$ Fig. $\mathbf{4 a}$. Thus, these results suggest that intragastric DKT produces a significant increase in GMBF via activation of TRPV1 and TRPA1, which are expressed in capsaicin-sensitive sensory nerves, especially in the late phase, but not in the gastric epithelial cells of anesthetized rats. Numerous TRPV1-immunoreactive axons were found around the arterioles in the submucosal layer of the stomach ( $>$ Fig. 6a). TRPA1-immunoreactive axons were also observed around the arterioles in the submucosal and circular muscle of the stomach ( $\triangleright$ Fig. 6 b). Additionally, TRPA1-immunoreactive cell bodies were also present in the myenteric plexus of the stomach. Interestingly, capsaicin-pretreatment to produce deafferentation of capsaicin-sensitive sensory fibers in rats abolished TRPA1-immunoreactivities in the submucosa but not in the circular muscle of the stomach ( Fig. 6d). However, TRPV1-immunoreactivities were abolished entirely in the submucosal and mucosal layers of the stomach ( Fig. 6c). Moreover, TRPA1-immunoreactive cells were also confirmed in the gastric mucous of both the control and capsaicin-pre- 


\section{Control}
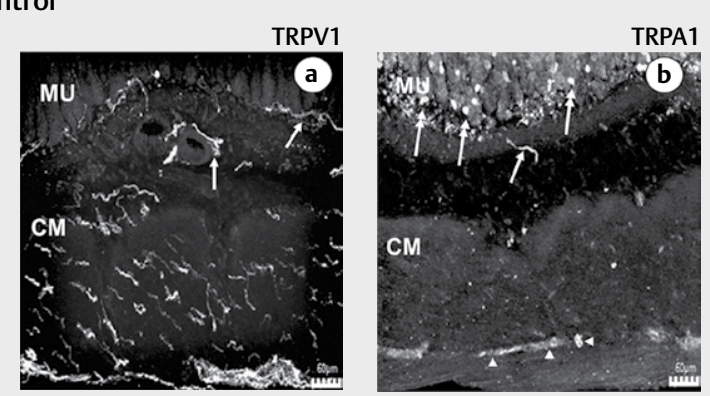

Capsaicin-pretreatment
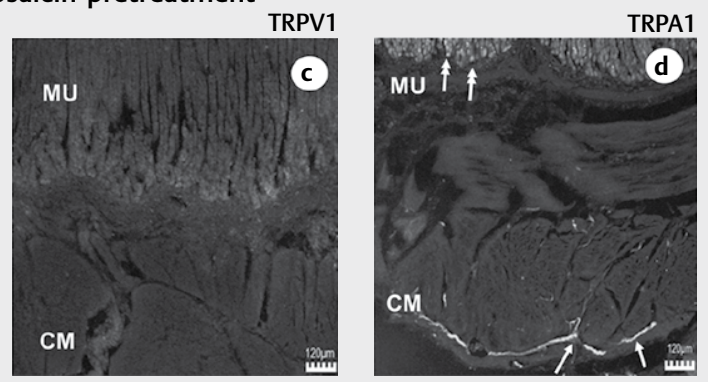

- Fig 6 Alteration in the distribution of immunoreactivities of TRPV1 and TRPA 1 in the cross-section of rat stomach in between control and capsaicin-pretreatment. The immunoreactive fibers of TRPV1 (a) and TRPA1 (b) were found in the submucosa, myenteric plexus, and muscularis. TRPV1- and TRPA1-expressing fibers were observed around the submucosal arteriole and venule bundles (arrows) (a, b). Note that TRPA1-immunoreactive cell bodies are present in the myenteric plexus (arrowheads), and the TRPA1-immunoreactivity was also confirmed in the mucous cells of the gastric glands (2-headed arrow). The scale bar is $60 \mu \mathrm{m}(\mathbf{a}, \mathbf{b})$. No TRPV1-expressing fiber is found in the capsaicinpretreated rat stomach (c). Notice that TRPA1-expressing fibers around the submucosal arteriole and venule bundles are not observed in capsaicin-pretreated rats (d), although the TRPA1-immunoreactivity is observed in the myenteric plexus (arrows) and mucous cells in the bottom of gastric glands after capsaicin pretreatment (2-headed arrow). MU, mucous membrane; CM, circular muscle. The scale bar corresponds to $120 \mu \mathrm{m}$ (c, d). Two transverse sections of tissues obtained from 5 normal and capsaicin-pretreated rats are stained by immunohistochemical methods. The typical photos are shown in this figure.

treatment groups. This was also present in the mucosal cell of the stomach after capsaicin-pretreatment ( Fig. $\mathbf{6 b}$ and d).

\section{Discussion}

In the present study, both the initial and late phases of increased GMBF in response to DKT were inhibited by the TRPA1 blocker A967079. However, only the late phase was inhibited by the TRPV1 blocker BCTC. In the capsaicin-pretreated rats, the late phase of the DKT-increased GMBF was abolished. Additionally, the immunohistochemical study clarified that TRPV1- and TRPA1-coexpressing nerve fibers were abolished in the submucosal layer in the capsaicin-pretreated rats. Taken together, these TRPV1- and TRPA1-coexpressing sensory nerve fibers are involved in the late phase of DKT-induced GMBF. Therefore, it was observed that an increase in
GMBF in response to DKT was significantly but not completely inhibited by the deafferentation of capsaicin-sensitive sensory fiber after capsaicin-pretreatment in the late phase (15-30 min) than in the initial phase $(0-15 \mathrm{~min})$.

In contrast, some myenteric TRPA1-expressing neurons in the smooth muscle layer remain in the capsaicin-pretreated rats, and they are thought to be the intrinsic primary afferent neurons. Therefore, it is supposed that those neurons that express TRPA1 but not TRPV1 are involved in the initial phase of DKT-induced GMBF. Interestingly, TRPA1-expressing mucosal cells were also observed in gastric glands. It is reported that the vasodilator Adrenomedullin is released from TRPA1-expressing intestinal epithelium in response to AITC and DKT in the rat intestine [8]. Therefore, the initial phase of DKT-induced GMBF may be attributed to vasodilators such as Adrenomedullin from the TRPA1-expressing mucosal cells. Taken together, the initial phase of increased GMBF in response to DKT may be due to the activation of TRPA1 that is expressed in sensory neurons and gastric epithelial cells.

It is well known that gastric hyperemic responses increase after the first application of capsaicin. However, the response to the second application of capsaicin is approximately one-third of that of the first application, suggesting that mucosal hyperemic responses are desensitized by repeated application of capsaicin $[9,10]$. Additionally, the repeated application of AITC induces desensitization of GMBF, suggesting that TRPA1 can be desensitized by AITC [11]. In contrast, the response to the second application of DKT was almost as strong as that to the first application (Supporting Information Fig. 1b), suggesting that the increased GMBF in response to DKT may be attributed to not only the activation of TRPV 1 and TRPA1 but also the blockage of 2-pore-domain potassium channels (KCNKs). For example, Zanthoxylum fruit was one of the ingredients of DKT, a known activator of TRPA1 and TRPV1 (actions resembling those of processed ginger compounds) and a blocker of the potassium leak channels, such as KCNKs [12]. Additionally, KCNKs maintain the cell's resting potential and regulate cellular excitability in exciting cells such as neurons. Therefore, DKT may be blocking KCNKs in capsaicin-sensitive sensory fibers by the action of Zanthoxylum fruit, which leads to increased membrane excitability. There is a decreased threshold for additional exogenous stimuli such as DKT [13]. Although it has been shown that capsaicin and AITC activated TRPV1 and TRPA1, respectively, they also inhibited nerve conduction via voltage-gated $\mathrm{Na}^{+}$channels in a manner independent of TRPV1 and TRPA 1 activation in the sciatic nerve of frogs $[14,15]$. The inhibition of nerve conduction required about 20-100 times higher concentration of capsaicin $(200 \mu \mathrm{M})$ and AITC $(2 \mathrm{mM})$ than the activation of TRPV1 $(1 \mu \mathrm{M})$ and TRPA $1(100 \mu \mathrm{M})$ in the DRG neurons [16, 17]. This study did not observe whether nerve conduction was inhibited by capsaicin and AITC, which might have increased GMBF. The increased GMBF in response to capsaicin and AITC was induced by TRPV1- and TRPA1-co-expressing sensory nerves.

It is shown that the TRPV1 blocker BCTC also inhibited the activation of TRPM8 in response to menthol in TRPM8-transfected HEK293 cells in vitro, so we studied whether or not the increased GMBF induced by mucosal application to the TRPM8 activator menthol $(100 \mathrm{mM})$ or WS-12 $(0.2-0.8 \mathrm{mM})$ was observed in rat stomachs [18]. However, it was not observed that menthol and WS-12 
induced the increased GMBF, suggesting that increased GMBF was not attributed to the activation TRPM8.

Previously, it has been reported that 1) numerous TRPV1-immunoreactive axons are found around the arteries in the lower mucosa of the rat stomach [19] and 2) capsaicin stimulates the TRPV1 nerve fibers to release CGRP [20] and nitric oxide (NO) [10,21]. Additionally, TRPV1 and TRPA 1 are co-expressed in the extrinsic sensory nerves [22]. These data led us to hypothesize that activating TRPA1- and TRPV1-expressing sensory nerves by DKT increased GMBF. Indeed, in this study, an increased GMBF induced by DKT in the deafferentation of capsaicin-sensitive sensory fibers in rats was mainly and strongly inhibited in the late phase. Further studies are required to elucidate the unknown vasorelaxant mediators to clarify the mechanism underlying the increased GMBF in response to DKT. Kampo medicines are used to treat various diseases in Japan. DKT is one of the most frequently used Kampo medicines, which is clinically effective in treating cold sensations and dysmotility in the abdomen. Although previous studies have shown the effects of DKT in improving these symptoms [1,5], our results indicate that DKT stimulates TRPV 1 and TRPA 1 on sensory nerves to promote gastric mucosal circulation.

In conclusion, the present study showed that the mucosal application of DKT increased GMBF via TRPA1- and TRPV1-expressing sensory nerves. For the first time, we demonstrated that the initial and the late phases of the increased GMBF in response to DKT were inhibited by the TRPA1 blocker A967079. However, only the late phase was inhibited by the TRPV1 blocker BCTC. In the capsaicinpretreated rats, the late phase of the DKT-increased GMBF was abolished. Additionally, the immunohistochemical study clarified that TRPV1- and TRPA1-coexpressing nerve fibers were abolished in submucosal layers in the capsaicin-pretreated rats. Taken together, these TRPV1- and TRPA1-coexpressing sensory nerve fibers are involved in the late phase of DKT-increased GMBF.

\section{Materials and Methods}

\section{Animals}

Male Sprague-Dawley rats weighing 180-220 g were purchased from Japan SLC Co., Ltd. Animals were housed under controlled environmental conditions (temperature: $24 \pm 2{ }^{\circ} \mathrm{C}$ and lights on from 7:00 am to 7:00 pm), and they were fed commercial rat chow MF (Oriental Yeast, Tokyo, Japan). The animals were kept in individual cages with raised mesh bottoms to prevent coprophagia and were deprived of food but allowed free access to tap water for $18 \mathrm{~h}$ before the experiments. Animal experiments were performed in compliance with the "Guiding Principles for the Care and Use of Laboratory Animals" approved by the Japanese Pharmacological Society. The study procedure was approved by the Ethical Committee on Animal Care and Animal Experimentation of Josai International University (\#1800051, registered on March 13, 2018, and \# 1900011, registered on March 1, 2019). A minimum number of animals were used for meaningful interpretation of data.

\section{Materials}

The following drugs were used: AITC (purity $>95 \%$, Tokyo Chemical Industry, Saitama, Japan), atenolol (purity >98\%, Wako, Osaka,
Japan), capsaicin (purity>60\%, Wako, Osaka, Japan), DL-isoproterenol (purity $>98 \%$, Wako, Osaka, Japan), urethane (ethyl carbamate, purity $>98 \%$, Wako, Osaka, Japan), A-967079 (purity $>98 \%$, Tocris Cookson, MN, USA), and BCTC (purity $>98 \%$, Biomol, PA, USA). AITC was suspended in $0.1 \%$ Tween 80 (purity $>75 \%$, Wako, Osaka, Japan)-saline for mucosal application. Capsaicin was dissolved in Tween 80-ethanol solution (10\% ethanol (Wako, Osaka Japan), $10 \%$ Tween 80 , and $80 \%$ saline (Otsuka, Tokushima, Japan) [23] for subcutaneous (s.c.) injection, or it was suspended in $0.5 \%$ CMC (Wako, Osaka, Japan) for mucosal application. DKT extract (Tsumura, Tokyo, Japan \& Co., Lot No. 2100100010) and maltose syrup powder (Tsumura, Tokyo, Japan \& Co., Lot No. 3020168) were used in this study. The composition of the herbal DKT medicine is the following: (1) Japanese pepper (Zanthoxylum fruit; peel of Zanthoxylum piperitum De Candolle), (2) processed ginger (Zingiberis Siccatum Rhizoma; rhizome of Zingiber officinale Roscoe), (3) ginseng (Ginseng radix; root of Panax ginseng C. A. Meyer), and (4) maltose syrup powder, which was used as glue. The traditional medicine was made by mixing the herbs in defined ratios by the Pharmacopoeia of Japan, followed by extraction using hot water. Finally, the extract was made into a powder form [12].

DKT extract powder was manufactured as an aqueous extract containing processed ginger, ginseng, and zanthoxylum fruit in a ratio of 5:3:2. HPLC analysis showed the presence of 6-gingesulfonic acid, hydroxy $\alpha$-sanshool, hydroxy $\beta$-sanshool, 6 -gingerol, $\alpha$-sanshool, $\beta$-sanshool, $\gamma$-sanshool, and 6 -shogaol. These are the main compounds of zanthoxylum fruit and dry-processed ginger. (Supporting Information Fig. S2). DKT was prepared by mixing DKT extract powder and maltose syrup powder in a ratio of 1:8. The DKT extract was suspended in distilled water for intragastric (i.g.) application. Although the doses of DKT in the present study $(1440 \mathrm{mg} /$ $\mathrm{mL}$ ) were higher than the clinical doses used in humans, previous studies in animals have shown that the relevant pharmacological effects occur only at the experimental doses. A-967079 was dissolved in DMSO (Wako) in saline, and the final concentration of DMSO was less than $5.0 \%$. Other drugs were dissolved in saline with no organic solvents or detergents. Drugs were administered at a volume of $0.5 \mathrm{~mL} / 100 \mathrm{~g}$ of body weight in the case of intraperitoneal (i.p.) and s.c. administration. Control animals received only the vehicle.

\section{GMBF measurement}

The animals were anesthetized with urethane $(1.25 \mathrm{~g} / \mathrm{kg}$, i.p.). The stomach was exposed through a midline incision, delivered to the abdominal surface by gentle traction on the spleen, and the pylorus was ligated. A 2-part lucite chamber was used to maintain ex vivo conditions of the gastric mucosa [24]. The 2 holes were cannulated to perfuse the mucosa with saline $(\mathrm{NaCl}, 154 \mathrm{mmol} / \mathrm{L})$ at $37^{\circ} \mathrm{C}$ at a $1 \mathrm{~mL} / \mathrm{min}$ flow rate. The lucite base was lowered over the animal, and the stomach was drawn through the center hole with forceps. Then, the stomach was opened along the greater curvature, and the edges were expanded by gently stretching the glandular mucosa. A plastic rim was then applied and pressed down onto the mucosa, such that only the glandular mucosa area was exposed [25]. The chamber was set at the level of the abdominal wall so that the external wall of the stomach remained inside the abdominal cavity. The body temperature was maintained at ap- 
proximately $37^{\circ} \mathrm{C}$ using an incandescent lamp. GMBF was measured using laser-Doppler flowmetry (Model ALF-21N, Advance, Tokyo, Japan) and a touching probe (diameter, $1 \mathrm{~mm}$ ) on the surface of the corpus mucosa. After stabilization of GMBF, the perfusion was discontinued, the luminal solution was removed, and mucosa was exposed to $2 \mathrm{~mL}$ of DKT for 10 or $30 \mathrm{~min}$. After applying DKT, the mucosa was rinsed with saline, another $2 \mathrm{~mL}$ of saline was instilled, and the perfusion was resumed. Changes in GMBF were continuously monitored and recorded for $2 \mathrm{~h}$ test periods using a Power Lab system (Model ML845; AD Instruments, Nagoya, Japan). Either the BCTC (2.7 mmol/L, i.g.) or A-967079 (10 mmol/L, i.g.) was given for $50 \mathrm{~min}$, starting at $20 \mathrm{~min}$ before DKT application $[26,27]$. Deafferentation of capsaicin-sensitive sensory fiber, which means capsaicin-pretreatment, was performed 2 weeks before the experiment by successive s.c. injections of capsaicin (20,30, and $50 \mathrm{mg} / \mathrm{kg}$ ) once daily for 3 days [23]. All capsaicin injections were given under ether anesthesia, and the rats were pretreated with the $\beta$-adrenergic receptor agonist isoproterenol $(0.01 \mathrm{mg} / \mathrm{kg}$, intramuscular (i.m.)) and the selective $\beta_{1}$-adrenergic antagonist atenolol $(0.01 \mathrm{mg} / \mathrm{kg}$, i.m. $)$ to counteract the respiratory impairment associated with capsaicin. To verify the effectiveness of the treatment, we placed a drop of capsaicin $(0.1 \mathrm{mg} / \mathrm{mL})$ in $0.5 \% \mathrm{CMC}$ into one eye of each rat, and the protective wiping movements were counted [28].

\section{Immunostaining}

The corpora of rat stomachs were fixed by immersing in $4 \%$ fresh paraformaldehyde in $0.1 \mathrm{~mol} / \mathrm{L}$ phosphate butter $\left(\mathrm{pH} \mathrm{7.4)}\right.$ at $4{ }^{\circ} \mathrm{C}$ for $2 \mathrm{~h}$. Subsequently, they were washed thrice with PBS and cryoprotected overnight in $0.1 \mathrm{~mol} / \mathrm{L}$ phosphate buffer containing $20 \%$ sucrose. The tissues were frozen in optimal cutting temperature (OCT) compound mounting medium (Sakura Finetek, Japan) and sectioned into $60 \mu \mathrm{m}$ thick sections using a cryostat (Leica, Hemel Hempstead, UK). The tissue sections were thawed and mounted onto MAS courting-slides (MSA-15, Matsunami Glass, Japan). Prior to staining, the slide-mounted sections were incubated in PBS containing $0.3 \%$ hydrogen peroxide (purity $=30 \%$, Wako, Osaka, Japan) for $30 \mathrm{~min}$ to quench endogenous peroxidase activity and washed with PBS. The sections were successively incubated in $10 \%$ normal donkey serum containing $0.2 \%$ Triton $\mathrm{X}-100,0.1 \%$ sodium azide (rehydration according to instruction to yield $100 \%$ serum, Jackson ImmuneResearch Laboratories, PA, USA) in PBS for $1 \mathrm{~h}$, and a polyclonal anti-TRPV1 antibody (1:30,000, Neuromics, MN, USA) or polyclonal anti-TRPA1 antibody (1:120,000; Abcam, Australia) for $40 \mathrm{~h}$ at $25^{\circ} \mathrm{C}$. TRPV 1 immunoreactivity was detected using a polyclonal antiserum raised in a rabbit against the carboxy terminus of mouse TRPV1. In contrast, TRPA1 immunoreactivity was detected using a polyclonal antiserum raised in rabbits against the fourth cytoplasmic loop of rat and mouse TRPA1. After washing with PBS, the sections were incubated with biotinylated donkey anti-rabbit immunoglobulin G (1:400; Jackson ImmunoResearch Laboratories, PA, USA) at room temperature for $90 \mathrm{~min}$. After further washes with PBS 3 times, the sections were incubated in streptavidin-biotincomplex (1:5 in PBS, Vectastain Elite ABC kit, Vector Laboratories, (A, USA) at room temperature for $1 \mathrm{~h}$, followed by incubation with fluorescein tyramide (1:75; TSA kit; Perkin Elmer Life Sciences, MA, USA). In control experiments, either the TRPV1 antibody or TRPA1 antibody was omitted from the staining procedure to verify staining specificity. No immunolabeling was observed in any of the controls. Each antiserum was diluted in PBS containing $0.2 \%$ Triton $\mathrm{X}-100$ and $0.1 \%$ sodium azide.

\section{Microscopy}

Sections were viewed on either a Leica epifluorescence microscope (Leica microsystems, Wetzlar, Germany) using Y3 (TRITC) and L4 (FITC) filter blocks or an upright Olympus confocal laser scanning microscope (FV-1000, Tokyo, Japan) system (FX-1000) with 20X and 40X Plan Neofluar objectives. A single tracking mode was used for single FITC labeling in the latter. Sections were scanned in the multi-tracking mode to avoid channel crosstalk when double labeling was performed with FITC ( $488 \mathrm{~nm})$ and TRITC (543 nm). Each image was produced by projecting all stack slices onto a single plane. The final plates were prepared using Adobe Photoshop.

\section{Statistical analysis}

The data are presented as the mean \pm SE for $4-8$ rats per group. Concentration-dependency was determined using analysis of variance. Statistical analysis was performed using Dunnett's multiple comparison test and Student's t-test on Sigma Plot 13.0 software (Systat) for Windows. Statistical significance was set at $\mathrm{p}<0.05$.

\section{Acknowledgments}

The authors are grateful to Tsumura Corporation for providing the DKT extract and its HPLC analysis. The authors also thank Marina Minamizawa, Imari Kawabe, and Ren Uchiki for performing animal experiments. This work was supported by a Grant-in-Aid for Scientific Research from the JSPS KAKENHI Grant Numbers JP17K08341 and JP18K06688, and by a subsidy from the research incentive system under the jurisdiction of the President of Josai International University. We would like to thank Editage (www.editage.com) for English language editing.

\section{Conflicts of Interest}

The authors declare that they have no conflict of interest.

\section{References}

[1] Manabe N, Camilleri M, Rao A, Wong B, Burton D, Busciglio L, Zinsmeister AR, Haruma K. Effect of daikenchuto (TU-100) on human gastrointestinal and colonic transit. Am J Physiol Gastrointest Liver Physiol 2010; 298: G970-G975

[2] Katsuno H, Maeda K, Kaiho T, Kunieda K, Funahashi K, Sakamoto J, Kono T, Hasegawa H, Furukawa Y, Imazu Y, Morita S, Watanabe M. Clinical efficacy of Daikenchuto for gastrointestinal dysfunction following colon surgery: a randomized, double-blind, multicenter, placebo-controlled study (JFMC39-0902). Jpn J Clin Oncol 2015; 45: 650-656

[3] Takayama S, Seki T, Watanabe M, Monma Y, Sugita N, Konno S, Iwasaki K, Takeda T, Yambe T, Yoshizawa M, Nitta S, Yaegashi N. The herbal medicine Daikenchuto increases blood flow in the superior mesenteric artery. Tohoku J Exp Med 2009; 219: 319-330 
[4] Kono T, Koseki T, Chiba S, Ebisawa Y, Chisato N, Iwamoto J, Kasai S. Colonic vascular conductance increased by daikenchuto via calcitonin gene-related peptide and receptor-activity modifying protein 1. J Surg Res 2008; 150: 78-84

[5] Satoh K, Kase Y, Hayakawa T, Murata P, Ishige A, Sasaki H. Dai-kenchuto enhances accelerated small intestinal movement. Biol Pharm Bull 2001; 24: 1122-1126

[6] Kikuchi D, Shibata C, Imoto H, Naitoh T, Miura K, Unno M. Intragastric Dai-Kenchu-To, a Japanese herbal medicine, stimulates colonic motility via transient receptor potential cation channel subfamily $\mathrm{V}$ member 1 in dogs. Tohoku J Exp Med 2013; 230: 197-204

[7] Mochiki E, Yanai M, Ohno T, Kuwano H. The effect of traditional Japanese medicine (Kampo) on gastrointestinal function. Surg Today 2010; 40: 1105-1111

[8] Kono T, Kaneko A, Omiya Y, Ohbuchi K, Ohno N, Yamamoto M. Epithelial transient receptor potential ankyrin 1 (TRPA1)-dependent adrenomedullin upregulates blood flow in rat small intestine. Am J Physiol 2013; 304: G428-G436

[9] Matsumoto J, Takeuchi K, Ueshima K, Okabe S. Role of capsaicin-sensitive afferent neurons in mucosal blood flow response of rat stomach induced by mild irritants. Dig Dis Sci 1992; 37: 1336-1344

[10] Raimura M, Tashima K, Matsumoto K, Tobe S, Chino A, Namiki T, Terasawa K, Horie S. Neuronal nitric oxide synthase-derived nitric oxide is involved in gastric mucosal hyperemic response to capsaicin in rats. Pharmacology 2013; 92: 60-70

[11] Wang S, Dai Y, Kogure Y, Yamamoto S, Zhang W, Noguchi K. Etodolac activates and desensitizes transient receptor potential ankyrin 1. Journal of Neurosci Res 2013; 91: 1591-1598

[12] Kono T, Shimada M, Yamamoto M, Kaneko A, Oomiya Y, Kubota K, Kase Y, Lee K, Uezono Y. Complementary and synergistic therapeutic effects of compounds found in Kampo medicine: analysis of daikenchuto. Front Pharmacol 2015; 6: Article 159 (p 1-7)

[13] Kubota K, Ohtake N, Ohbuchi K, Mase A, Imamura S, Sudo Y, Miyano $\mathrm{K}$, Yamamoto M, Kono T, Uezono Y. Hydroxy- $\alpha$ sanshool induces colonic motor activity in rat proximal colon: a possible involvement of KCNK9. Am J Physiol 2015; 308: G579-G590

[14] Tomohiro D, Mizuta K, Fujita T, Nishikubo Y, Kumamoto E. Inhibition by capsaicin and its related vanilloids of compound action potentials in frog sciatic nerves. Life Sci 2013; 92: 368-378

[15] Matsushita A, Ohtsubo S, Fujita T, Kumamoto E. Inhibition by TRPA1 agonists of compound action potentials in the frog sciatic nerve. Biochem Biophys Res Commun 2013; 434: 179-184

[16] Davis JB, Gray J, Gunthorpe MJ, Hatcher JP, Davey PT, Overend P, Harries MH, Latcham J, Clapham C, Atkinson K, Hughes SA, Rance K, Grau E, Harper AJ, Pugh PL, Rogers DC, Bingham S, Randall A, Sheardown SA. Vanilloid receptor- 1 is essential for inflammatory thermal hyperalgesia. Nature 2000; 405: 183-187
[17] Kosugi M, Nakatsuka T, Fujita T, Kuroda Y, Kumamoto E. Activation of TRPA1 channel facilitates excitatory synaptic transmission in substantia gelatinosa neurons of the adult rat spinal cord. J Neurosci 2007; 27: 4443-4451

[18] Behrendt HJ, Germann T, Gillen C, Hatt H, Jostock R. Characterization of the mouse cold-menthol receptor TRPM8 and vanilloid receptor type-1 VR1 using a fluorometric imaging plate reader (FLIPR) assay. $\mathrm{Br}$ J Pharmacol 2004; 141: 737-745

[19] Horie S, Yamamoto H, Michael G], Uchida M, Belai A, Watanabe K, Priestley JV, Murayama T. Protective role of vanilloid receptor type 1 in $\mathrm{HCl}$-induced gastric mucosal lesions in rats. Scand J Gastroenterol 2004; 39: 303-312

[20] Horie S, Michael G], Priestley JV. Co-localization of TRPV1-expressing nerve fibers with calcitonin-gene-related peptide and substance $P$ in fundus of rat stomach. Inflammopharmacology 2005; 13: 127-137

[21] Horie S, Raimura M, Matsumoto K, Namiki T, Terasawa K, Priestley JV, Tahima K. Cooperative effects of neuronal nitric oxide synthase and endothelial nitric oxide synthase on gastric hyperemic response to intragastric capsaicin. In: Mozsik G, Abdel-Salam OME, Takeuchi K, eds. Capsaicin-Sensitive Neural Afferentation and the Gastrointestinal Tract: From Bench To Bedside 2014; 4: 103-124

[22] Kondo T, Obata K, Miyoshi K, Sakurai J, Tanaka J, Miwa H, Noguchi K. Transient receptor potential A1 mediates gastric distention-induced visceral pain in rats. Gut 2009; 58: 1342-1352

[23] Esplugues JV, Ramos EG, Gil L, Esplugues ]. Influence of capsaicinsensitive afferent neurons on the acid secretory responses of the stomach in vivo. Br J Pharmacol 1990; 100: 491-496

[24] Takeuchi K, Ishihara Y, Okada M, Niida H, Okabe S. A continuous monitoring of mucosal integrity and secretory activity in rat stomach: a preparation using a lucite chamber. Jpn J Pharmacol 1989; 49: 235-244

[25] Tashima K, Nakashima M, Kagawa S, Kato S, Takeuchi K. Gastric hyperemic response induced by acid back-diffusion in rat stomachs following barrier disruption-relation to vanilloid type- 1 receptors. Med Sci Monit 2002; 8: BR157-BR163

[26] Christoph T, Gillen C, Mika J, Grünweller A, Schäfer MK, Schiene K, Frank R, Jostock R, Bahrenberg G, Weihe E, Erdmann VA, Kurreck J. Antinociceptive effect of antisense oligonucleotides against the vanilloid receptor VR1/TRPV1. Neurochem Int 2007; 50: 281-290

[27] Pomonis JD, Harrison JE, Mark L, Bristol DR, Valenzano KJ, Walker K. $\mathrm{N}$-(4-Tertiarybutylphenyl)-4-(3-cholorphyridin-2-yl) tetrahydropyrazine-1 (2H)-carbox-amide (BCTC), a novel, orally effective vanilloid receptor 1 antagonist with analgesic properties: II. in vivo characterization in rat models of inflammatory and neuropathic pain. J Pharmacol Exp Ther 2003; 306: 387-393

[28] Yonei Y, Holzer P, Guth PH. Laparotomy-induced gastric protection against ethanol injury is mediated by capsaicin-sensitive sensory neurons. Gastroenterology 1990; 99: 3-9

\section{Notice}

This article was changed according to the following Erratum on June $15^{\text {th }} 2022$.

\section{Erratum}

In the above mentioned article the page numbers were corrected in e72 ff. 